\title{
Biomasa de Eucalyptus nitens de 4-7 años de edad en un rodal de la X Región, Chile
}

\author{
Biomass of 4-7 year-old Eucalyptus nitens in a stand in the X Region, Chile
}

\author{
Edith Geldres ${ }^{1}$, Víctor Gerding ${ }^{2 *}$, Juan E Schlatter ${ }^{2}$ \\ ${ }^{1}$ Universidad de Los Lagos, Departamento de Ciencia y Tecnología Forestal. \\ *Autor de correspondencia: ${ }^{2}$ Universidad Austral de Chile, Facultad de Ciencias Forestales, casilla 567, \\ Valdivia, Chile, Tel.: 56-63-221431, vgerding@uach.cl
}

\begin{abstract}
SUMMARY
The total biomass of a Eucalyptus nitens plantation was estimated in age sequence from 4 to 7 years, with fertilization (X Region, $\left.41^{\circ} 01^{\prime} S-73^{\circ} 27^{\prime} \mathrm{W}\right)$, in red clayey soil of Crucero series. The forest biomass at ages 4, 5 and 7 was estimated from the corresponding average quadratic diameter (DMC) of the tree; being interpolated at age 6. Components of the crown, stem and roots were obtained; additionally humus and litter were calculated. The total biomass of the stand reached $92 \mathrm{t} \mathrm{ha}^{-1}$ at $4 \mathrm{years} 131 \mathrm{t} \mathrm{ha} \mathrm{e}^{-1}$ at 5 years and $181 \mathrm{t} \mathrm{ha}^{-1}$ at 6 years with a density of 1,500 trees ha $\mathrm{h}^{-1}$. After thinning, between ages 6-7, the total biomass increase was $29.8 \mathrm{t} \mathrm{ha}^{-1}$. Yearly root increase was $0.5,2.4$ and $3.8 \mathrm{t} \mathrm{ha}^{-1}$ in the sequence $4-7$ years old. Thinning provided the soil with $21.7 \mathrm{t} \mathrm{ha}^{-1}$ of waste (crowns and bark) and exported $49 \mathrm{t} \mathrm{ha}^{-1}$ of wood. Pruning of the lowest third of crowns $\left(800\right.$ trees ha- $\mathrm{h}^{-1}$ ) at 5 years contributes with $5.8 \mathrm{t} \mathrm{ha}^{-1}$ of branches and $3.8 \mathrm{t} \mathrm{ha}^{-1}$ of leaves to the soil.
\end{abstract}

Key words: Eucalyptus nitens, biomass, roots, humus, litter.

\section{RESUMEN}

Se estimó la biomasa total de una plantación de Eucalyptus nitens en una secuencia de edad de 4 a 7 años, con fertilización (X Región, $41^{\circ} 01^{\prime} S-73^{\circ} 27^{\prime}$ O), en un suelo rojo arcilloso de la serie Crucero. La biomasa del bosque en las edades de 4,5 y 7 años fue estimada a partir del respectivo árbol de diámetro medio cuadrático; a los 6 años fue interpolada. Se obtuvieron componentes de la copa, tronco y raíces; además se midió mantillo y hojarasca. La biomasa total del rodal alcanzó a 92 t ha ${ }^{-1}$ a los 4 años, 131 a los 5 años y 181 a los 6 años, con una densidad de 1.500 árboles ha ${ }^{-1}$. Posterior al raleo, entre los 6-7 años,

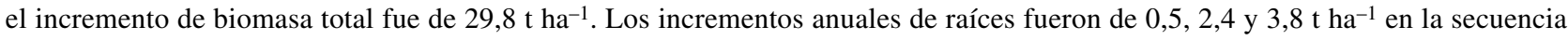
4-7 años. El raleo aportó al suelo $21,7 \mathrm{t} \mathrm{ha}^{-1}$ de desechos (copas y corteza) y exportó $49 \mathrm{t} \mathrm{ha}^{-1}$ de madera. La poda del tercio

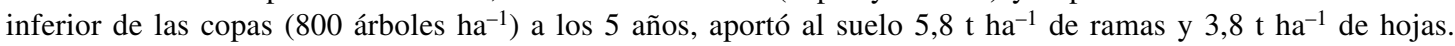

Palabras clave: Eucalyptus nitens, biomasa, raíces, mantillo, hojarasca.

\section{INTRODUCCIÓN}

La Décima Región presenta alrededor de seiscientas mil hectáreas factibles de ser plantadas con especies forestales de rápido crecimiento, entre las cuales Eucalyptus nitens (Dean et Maiden) Maiden es una de las que tiene mejores posibilidades de adaptación y adecuado valor comercial de su madera. Una de las ventajas de E. nitens es su resistencia a las bajas temperaturas (FAO 1981, Prado y Barros 1989, Eldridge et al. 1994), como las que ocurren en esta región. El rápido crecimiento de la especie, sin embargo, plantea un desafío silvicultural, requiriendo de esquemas de manejo adecuados a su ritmo de crecimiento y de las precauciones necesarias para asegurar una producción sostenible de biomasa en rotaciones cortas y sucesivas. En Chile existen sólo datos aislados de crecimiento de E. nitens, y más escasos aún los que se refieren a sus demandas nutritivas en cada una de sus etapas de crecimiento. En la Décima Región no se cuenta con curvas de crecimiento de biomasa de E. nitens, lo que dificulta lograr lo anteriormente expuesto y conocer el balance nutritivo de estas plantaciones y, por ende, no hay claridad del efecto de la especie sobre el estado nutricional del suelo. Por este motivo, existe la necesidad de conocer la biomasa acumulada de la especie a través del tiempo para determinar curvas de crecimiento de biomasa $\mathrm{y}$, posteriormente, de demanda de elementos nutritivos. 
Existe una biomasa óptima naturalmente sustentable para cada sitio que depende de la disponibilidad de nutrientes, precipitaciones, capacidad de retención de agua del suelo y de las práctica de manejo del sitio (Noble y Herbert 1989). De igual modo, el balance nutricional del sitio depende de la interacción de la especie en cuestión con factores del suelo, del clima y del manejo silvicultural (Gerding y Schlatter 1999, Schlatter y Gerding 1999, Bonomelli y Suárez 1999). Para establecer los requerimientos nutricionales de una especie forestal es necesario estimar su potencial productivo (acumulación de biomasa), su balance nutricional en el sitio a través de las entradas y salidas de elementos nutritivos desde el rodal, como también su redistribución interna, los que pueden ser conocidos a través de los flujos y acumulación de la materia orgánica (De Freitas et al. 1995, Grove et al. 1996, Judd et al. 1996).

Respecto a la biomasa aérea de plantaciones de E. nitens creciendo en distintos sitios en Chile, se han registrado crecimientos medios anuales de 10,8 a 24,4 t ha-1 en edades de 4 a 7 años (Bonomelli y Suárez 1999, Aparicio 2001, Gerding et al. 2002, Muñoz 2002, Toro 2002, Saavedra 2005). Este crecimiento de E. nitens en Chile es equivalente o superior al observado en otros países para esta especie y al de otros eucaliptos como E. saligna Sm., E. regnans F. Muell. y E. fastigata Dean et Maiden (Attiwill y Leeper 1987, Madgwick et al. 1981, Frederick et al. 1985).

A medida que se desarrolla la especie, la distribución porcentual de los distintos componentes de la biomasa aérea registran un cambio natural con la edad, aumentando la proporción de madera, disminuyendo la de las ramas y hojas, mientras que la corteza después de algunos años se mantiene relativamente constante (Bonomelli y Suárez 1999, Aparicio 2001, Saavedra 2005). Sitios de diferente productividad presentan similar distribución de componentes en la biomasa y la aplicación de fertilizantes no ha mostrado incidencia en dicha distribución. En esto E. nitens ha mostrado un comportamiento similar al de E. globulus Labill. (Garcinuño 1995).

Con respecto al reciclado de elementos nutritivos, la cantidad de ellos en las hojas caídas está dada por su biomasa y su contenido. Por lo tanto, si se conoce la tasa anual de acumulación de los componentes de la biomasa de los árboles, es posible estimar para un año determinado el reciclaje o ciclo externo que representan las hojas (Bonomelli et al. 2002). En E. nitens de 3 a 6 años de edad se han observado producciones anuales de hojarasca de 4 a $9 \mathrm{t} \mathrm{ha}^{-1}$, lo que representa una alta tasa de desechos para el reciclaje (Frederick et al. 1985, Aparicio 2001, Bonomelli et al. 2002). Esta caída de hojarasca ocurre principalmente durante los meses de mayor estrés hídrico, es decir, de preferencia en verano.

El presente trabajo tiene como propósito estimar la biomasa total de un rodal de Eucalyptus nitens en un periodo de edad de 4-7 años. Los objetivos específicos son: a) estimar la producción de biomasa aérea, radicular, de hojarasca (desechos) y la acumulación del mantillo; b) determinar el efecto de un raleo a los 5 años sobre la exportación y redistribución de la biomasa; y c) cuantificar el efecto de una poda a los 5 años sobre el reciclado de biomasa.

\section{MÉTODOS}

Caracterización del ensayo. El ensayo fue establecido en el predio Pichimaule de propiedad de Pürstinger-Ludwig y Cía. Ltda., situado a una altitud aproximada de 200 m s.n.m en la precordillera costera de la Décima Región, provincia de Llanquihue, en el noroeste de la comuna de Fresia $\left(41^{\circ} 01^{\prime} S-73^{\circ} 27^{\prime} O\right)$. De acuerdo a la clasificación Koeppen, el clima es de costa occidental con influencia mediterránea (Donoso 1992). Existe una frecuencia de heladas de 20-30 por año, con un periodo libre de heladas en el año de 150-200 días y una temperatura mínima de $-6^{\circ} \mathrm{C}$. El régimen de humedad anual es moderado, con una precipitación de 1.600-2.000 mm anuales, lo que marca un excedente para el consumo de la vegetación, no obstante en verano aumenta la evapotranspiración, por lo cual se presenta un déficit hídrico (Schlatter et al. 1995). El suelo es un rojo arcilloso de la serie Crucero (Acrudoxic Hydric Hapludand) originado de cenizas volcánicas depositadas sobre sedimentos fluvioglaciales (CIREN 2001).

El ensayo fue establecido en 1996 con una fertilización preplantación con roca fosfórica (Bifox), la cual aporta fósforo de baja solubilidad en agua, lo que disminuye el fenómeno de fijación inicial rápida, propia de estos suelos ácidos, favoreciendo el suministro de fósforo a mediano plazo. El esquema de fertilización incluyó superfosfato triple como fuente de fósforo soluble de rápida disponibilidad. Este esquema de fertilización se determinó en consideración a que el suelo presentaba como principal factor limitante un régimen de elementos nutritivos con bajos niveles de fósforo disponible (2-4 mg kg-1), presentaba alta acidez ( $\mathrm{pH}$ al agua 5,1) y elevada saturación de aluminio (35\%). El esquema de preparación del suelo y fertilización fue el siguiente en una superficie de 50 × $50 \mathrm{~m}\left(2.500 \mathrm{~m}^{2}\right)$ :

- Preplantación se controló la maleza con herbicida en todo el ensayo, se realizó subsolado a $50 \mathrm{~cm}$ en la hilera de plantación y allí se aplicó la roca fosfórica equivalente a $49 \mathrm{~kg} \mathrm{ha}^{-1}$ de fósforo.

- En octubre de 1996 se fertilizó con $135 \mathrm{~g} \mathrm{planta}^{-1}$ de NPK (20:25:5), incorporados en dos bandas de $40 \mathrm{~cm}$ de largo y $5 \mathrm{~cm}$ de profundidad, a $5-10 \mathrm{~cm}$ de las plantas. Esta fertilización correspondió a $36 \mathrm{~kg} \mathrm{ha}^{-1}$ de nitrógeno (urea), 19,9 de fósforo (superfosfato triple) y 5,6 de potasio (sulfato de potasio); la mezcla incluyó los micronutrientes zinc y cobre (sulfatos) y boro (boronatrocalcita). 
- En septiembre de 1997 se fertilizó al voleo en fajas junto a las hileras de árboles con $45 \mathrm{~kg} \mathrm{ha}^{-1} \mathrm{de}$ nitrógeno (urea), 48 de fósforo (superfosfato triple) y 10 de potasio (muriato), 4,8 de boro (boronatrocalcita), 1,25 de cobre (sulfato) y 1,15 de zinc (sulfato).

- En agosto de 1999 se fertilizó al voleo en toda la superficie del rodal con $230 \mathrm{~kg} \mathrm{ha}^{-1}$ de nitrógeno (supernitro) y 100 de fósforo (superfosfato triple).

Inventario forestal. Las plantaciones se establecieron con un espaciamiento de árboles aproximado de $4 \mathrm{~m}$ entre hileras y $1,5 \mathrm{~m}$ en la hilera (Leiva 2000, Staub 2000, Aparicio 2001). Se consideró una densidad residual de 1.500 árboles $\mathrm{ha}^{-1}$ a las edades de 4 y 5 años. Posteriormente, cumplidos los 5 años de la plantación, se practicó un raleo que dejó una densidad residual de 800 árboles ha- ${ }^{-1}$, densidad que se mantuvo a los 6 y 7 años de edad. Los inventarios dasométricos anuales de la plantación permitieron el cálculo del diámetro medio cuadrático (DMC) considerando DAP $>5 \mathrm{~cm}$ (cuadro 1). La estimación del diámetro (DAP) de los árboles extraídos se obtuvo a partir de una relación funcional entre el DAP y el diámetro de tocón (DAT):

$$
\text { DAP }=0,8752 \text { DAT-0,7634 }\left(\mathrm{R}^{2}=92,61 \%, P<0,01\right) .
$$

Biomasa aérea y su distribución. La biomasa aérea por árbol se obtuvo según el siguiente procedimiento (Aparicio 2001, Geldres 2005a, Saavedra 2005): a los 4 y 5 años de edad del rodal, se seleccionaron 10 árboles, dos de cada una de las siguientes cinco clases de tamaño: DMC y DMC \pm 1 y \pm 2 desviaciones estándar; a los 7 años de edad se seleccionaron sólo 6 árboles, considerando dos árboles del DMC y uno en cada una de las otras clases diamétricas. Esto último se hizo en atención a la homogeneidad de la distribución de la biomasa en árboles de todo el rango diamétrico considerado y a la muy significativa estimación de biomasa aérea a partir

Cuadro 1. Valores dasométricos en la plantación de Eucalyptus nitens.

Dasometric values in the Eucalyptus nitens plantation.

\begin{tabular}{crc}
\hline $\begin{array}{c}\text { Edad } \\
\text { (años) }\end{array}$ & Densidad (árboles ha $\left.{ }^{-1}\right)$ & $\begin{array}{c}\text { DMC } \\
(\mathrm{cm})\end{array}$ \\
\hline 4 & 1.500 & 12,7 \\
5 & 1.500 & 15,0 \\
6 & $1.500 \mathrm{a}$ & 17,2 \\
6 & $800 \mathrm{~b}$ & 17,5 \\
6 & $700 \mathrm{c}$ & 16,7 \\
7 & 800 & 19,6
\end{tabular}

a: $\sin$ raleo ; b: densidad residual; c: densidad extraída del DAP y altura de los árboles (Aparicio 2001, Minte 2004, Saavedra 2005). La recolección de muestras de biomasa se realizó en mayo de 2001, 2002 y 2003. Los árboles talados se segregaron en componentes de la copa y el tronco: hojas juveniles, hojas adultas, ramillas ( $\leq 1 \mathrm{~cm}$ de diámetro), ramas ( $>1 \mathrm{~cm}$ de diámetro), ramas muertas, madera y corteza.

Los componentes de la copa fueron separados en tercios: inferior, medio y superior. En terreno se determinó el peso en húmedo de cada componente y se obtuvo una muestra de por lo menos un $20 \%$ de su peso total, en la cual se separaron los componentes. Posteriormente, de tales muestras se obtuvo el contenido de humedad (peso seco a $75^{\circ} \mathrm{C}$ ).

A los 4 años el fuste se fraccionó en tercios y a los 5 y 7 años se trozó cada $1 \mathrm{~m}$ y para obtener su peso húmedo. El contenido de humedad y la proporción de madera y corteza se obtuvieron de rodelas con corteza, de un grosor de 8-10 cm, extraídas desde la base y cada $2 \mathrm{~m}$. En cada rodela se midió diámetro con y sin corteza en el extremo basal.

La biomasa aérea promedio de los árboles de DMC fue estimada a través de funciones a las edades de 4, 5 y 7 años de la plantación. Para la edad de 6 años se ajustó un modelo de estimación de biomasa aérea en función de la edad (cuadro 2).

La distribución porcentual de la biomasa aérea a la edad de 6 años se estimó en función de la edad, considerando los valores registrados para las edades de 4,5 y 7 años de la plantación (cuadros 3 y 4). Excepcionalmente, el porcentaje de corteza no mostró una asociación con la edad, por lo que se estimó mediante la diferencia respecto del total.

Con la biomasa aérea por árbol representante del DMC (cuadro 2) se calculó la biomasa de los componentes aéreos de acuerdo a su distribución porcentual según la edad (cuadros 3 y 4). Posteriormente se obtuvo la biomasa aérea total por hectárea al multiplicar la biomasa del árbol DMC por la densidad del rodal y con ello también se obtuvieron los incrementos anuales respectivos.

Cuadro 2. Funciones de estimación de biomasa aérea de Eucalyptus nitens.

Functions of aerial biomass estimation of Eucalyptus nitens.

\begin{tabular}{|c|c|c|c|}
\hline Edad (años) & Función & $\mathrm{n}$ & $\mathrm{R}^{2}(\%)$ \\
\hline $4^{\mathrm{a}}$ & $\mathrm{B}=0,1508 \mathrm{D}^{2,2732}$ & 10 & $98,84 * *$ \\
\hline 5 & $\mathrm{~B}=0,1044 \mathrm{D}^{2,4261}$ & 10 & $97,56 * *$ \\
\hline 6 & $\mathrm{~B}=3,344 \mathrm{E}^{1,9306}$ & 3 & $100 * * *$ \\
\hline 7 & $\mathrm{~B}=0,2161 \mathrm{D}^{2,1836}$ & 6 & $99,65 * *$ \\
\hline
\end{tabular}


Cuadro 3. Distribución de la biomasa aérea (\%) de Eucalyptus nitens según componentes para árboles del DMC de 4-7 años de edad (coeficiente de variación en porcentaje entre paréntesis).

Aerial biomass distribution (\%) of Eucalyptus nitens according to tree components at ages 4-7 years (coefficient of variation between brackets).

\begin{tabular}{cccccccc}
\hline $\begin{array}{c}\text { Edad } \\
\text { (años) }\end{array}$ & Madera & Corteza & Hojas & $\begin{array}{c}\text { Ramas } \\
\text { vivas }\end{array}$ & Ramillas & $\begin{array}{c}\text { Ramas } \\
\text { muertas }\end{array}$ & Total \\
\hline 4 & $59,4(3,0)$ & $10,5(12,8)$ & $13,2(8,9)$ & $12,4(8,0)$ & - & $4,4(15,6)$ & 100 \\
5 & $63,1(3,3)$ & $8,4(5,9)$ & $12,8(13,2)$ & $6,3(36,5)$ & $5,4(19,3)$ & $3,9(8,0)$ & 100 \\
6 & $69,3-$ & $9,2-$ & $9,4-$ & $5,4-$ & $4,0-$ & 2,7 & - \\
100 & - & $2,7(13,8)$ & $1,6(54,2)$ & 100 \\
7 & $74,9(2,9)$ & $9,2(11,4)$ & $6,8(7,3)$ & $4,7(43,9)$ & $2,8)$ \\
\hline
\end{tabular}

- Sin registro. A los 6 años no hubo información para el coeficiente de variación.

Cuadro 4. Distribución de la biomasa de la copa (\%) en Eucalyptus nitens según componentes para árboles del DMC de 4-7 años de edad.

Crown biomass distribution (\%) of Eucalyptus nitens according to tree components at ages 4-7 years.

\begin{tabular}{clrcrc}
\hline $\begin{array}{c}\text { Edad } \\
\text { (años) }\end{array}$ & Tercio & Hojas & Ramillas & $\begin{array}{c}\text { Ramas } \\
\text { vivas }\end{array}$ & $\begin{array}{c}\text { Ramas } \\
\text { muertas }\end{array}$ \\
\hline \multirow{2}{*}{4} & Superior & 21,5 & - & 18,9 & 0 \\
& Medio & 61,9 & - & 61,4 & 0 \\
& Inferior & 16,6 & - & 19,7 & 100 \\
\hline \multirow{2}{*}{5} & Superior & 9,1 & 19,5 & 5,2 & 0 \\
& Medio & 42,0 & 36,2 & 38,4 & 0 \\
& Inferior & 48,9 & 44,3 & 56,3 & 100 \\
\hline \multirow{2}{*}{7} & Superior & 17,5 & 24,0 & 6,1 & 0 \\
& Medio & 40,4 & 35,6 & 28,5 & 0 \\
& Inferior & 42,2 & 40,4 & 65,4 & 100 \\
\hline
\end{tabular}

- Se registraron junto con las ramas vivas.

Biomasa radicular. Las raíces se obtuvieron de seis columnas de 10 x $10 \mathrm{~cm}$ de base y $110 \mathrm{~cm}$ de profundidad, considerando dos repeticiones en la línea de plantación, dos a $1 \mathrm{~m}$ y dos a $2 \mathrm{~m}$ de ella. Las raíces se separaron en finas $(\varnothing \leq 2 \mathrm{~mm})$ y gruesas $(\varnothing>2 \mathrm{~mm})$; no se consideró la raíz principal de los árboles en esta parte del muestreo. Se lavaron con agua para extraer el máximo posible de suelo adherido y se secaron a $75^{\circ} \mathrm{C}$ (Aparicio 2001, Moldenhauer 2003). Se obtuvo la biomasa promedio y su coeficiente de variación considerando las tres posiciones horizontales del muestreo. Para el año 6 la biomasa de raíces finas y gruesas se estimó como un promedio entre los registros de los 5 y 7 años de edad. La biomasa de la raíz principal para la edad de 4 años se obtuvo de Aparicio (2001). En el año 7 se determinó la biomasa de la raíz principal de tres árboles para estimar su equivalente en los árboles de DMC a las edades de 5, 6 y 7 años.

Biomasa del mantillo. El mantillo se obtuvo cada año a partir de nueve muestras de $0,1 \mathrm{~m}^{2}$, considerando tres posiciones: en la línea de plantación (A), a un metro (B) y a dos metros de esta línea (C) (Geldres 2005a). En las edades de 6 y 7 años se registró separadamente la biomasa de la corteza y de ramas producto del manejo (raleo y poda). El material fue secado a $75^{\circ} \mathrm{C}$.

Hojarasca. La recolección mensual de hojarasca se realizó en cajones de $50 \mathrm{~cm}$ x $50 \mathrm{~cm}$, con fondo de malla plástica, ubicados a 0,2 m desde el suelo (seis cajones en 2000 y 2001, nueve cajones en 2002 y 2003). El material fue secado a $75^{\circ} \mathrm{C}$. La biomasa de la hojarasca se acumuló por estaciones del año. El coeficiente de variación se calculó, para cada año, sobre la biomasa anual de hojarasca en cada posición horizontal.

\section{RESULTADOS}

Biomasa del rodal. Para una densidad de 1.500 árboles $\mathrm{ha}^{-1}$ la biomasa total (aérea y radicular) acumulada a los 4 años de edad fue de 91,6 $\mathrm{t} \mathrm{ha}^{-1}$, a los 5 años de 130,8 $\mathrm{t} \mathrm{ha}^{-1}$ y a $\operatorname{los} 6$ años de 180,9 $\mathrm{t} \mathrm{ha}^{-1}$. Esto equivale a incrementos anuales de biomasa de $39,2 \mathrm{t} \mathrm{ha}^{-1}$ en el periodo de 4 a 5 años de edad y de $50,1 \mathrm{t} \mathrm{ha}^{-1}$ en el periodo de 5 a 6 años de edad. A los 6 años de edad, para una densidad de 800 árboles ha $^{-1}$, producto del raleo, se registró una biomasa total acumulada de 110,1 t ha-1 y sobre igual densidad a los 7 años de edad se obtuvieron 139,9 $\mathrm{t} \mathrm{ha}^{-1}$. El incremento anual de biomasa total en este periodo fue de $29,8 \mathrm{t} \mathrm{ha}^{-1}$.

La acumulación de biomasa aérea con una densidad de 1.500 árboles ha ${ }^{-1}$ a los 4,5 y 6 años de edad fue de $73,1 \mathrm{t} \mathrm{ha}^{-1}, 111,8 \mathrm{t} \mathrm{ha}^{-1}$ y $159,5 \mathrm{t} \mathrm{ha}^{-1}$ respectivamente. La acumulación de la biomasa radicular también fue ascendente pero fluctuó en magnitudes muy inferiores, entre 18,5 $\mathrm{t} \mathrm{ha}^{-1}$ a los 4 años de edad y $21,4 \mathrm{t} \mathrm{ha}^{-1}$ a los 6 años. El incremento corriente anual de biomasa aérea presentó mayor variación porcentual que el de la biomasa radicular; este incremento en la biomasa aérea fue de $52,9 \%$ entre los 4 y los 5 años de edad y de $42,7 \%$ de los 5 a los 6 años. En cambio, la biomasa radicular registró un incremento muy pequeño de sólo $2,7 \%$ entre los 4 y 5 años, pero de $15,7 \%$ entre los 5 y 6 años. 
Posteriormente, bajo la condición raleada (800 árboles $\mathrm{ha}^{-1}$ ), el rodal acumuló una biomasa aérea de $88,7 \mathrm{t} \mathrm{ha}^{-1}$ a los 6 años y de $114,7 \mathrm{t} \mathrm{ha}^{-1}$ a $\operatorname{los} 7$ años (incremento de 29,3\%), con un equivalente de biomasa radicular de $21,4 \mathrm{t} \mathrm{ha}^{-1} \mathrm{y}$ de $25,2 \mathrm{t} \mathrm{ha}^{-1}$, respectivamente (incremento de $17,8 \%$ ).

El porcentaje de biomasa de raíces, con respecto al total de biomasa que acumuló el rodal, disminuyó de un $20 \%$ a un $12 \%$ con el aumento de la edad, desde los 4 hasta los 6 años. Consecuentemente, la proporción de biomasa aérea aumentó de 80 a $88 \%$ en igual periodo. Después del raleo el porcentaje de biomasa radicular fluctuó entre 18 y 19\% (6 y 7 años de edad).

En el periodo de edad de 4 a 6 años la biomasa de la madera del tronco aumentó de 43 a $70,1 \mathrm{t} \mathrm{ha}^{-1}$ en el primer año (incremento anual de $27,1 \mathrm{t} \mathrm{ha}^{-1}$ ) y luego el incremento fue de $40 \mathrm{t} \mathrm{ha}^{-1}$ en el segundo año. La biomasa de corteza también registró incrementos ascendentes: 1,7 y 5,3 t ha ${ }^{-1}$, respectivamente. En cambio, los incrementos corrientes anuales de la biomasa de la copa leñosa y de las hojas disminuyeron con la edad de la plantación. En el periodo de edad de 4 a 6 años la copa leñosa presentó incrementos anuales de $5,2 \mathrm{t} \mathrm{ha}^{-1}$ y $1,9 \mathrm{t} \mathrm{ha}^{-1}$ respectivamente, y las hojas de 4,7 a $0,7 \mathrm{t} \mathrm{ha}^{-1}$.

La biomasa de las hojas, las ramas y las ramillas se concentró en el tercio medio de la copa a la edad de 4 años pero, a medida que aumentó la edad del rodal, estas proporciones fueron mayores en el tercio inferior del árbol. Las ramas muertas, para todas las edades del rodal, sólo se observaron en el tercio inferior (cuadro 5).

Luego del raleo, entre los 6 y 7 años de edad, no se registró incremento de la copa en el rodal. Sin embargo, el incremento en biomasa de madera fue de $24,4 \mathrm{t} \mathrm{ha}^{-1}$, menor que antes del raleo, debido a la disminución de densidad. El porcentaje de biomasa de madera aumentó naturalmente con la edad del rodal, en cambio los porcentajes de biomasa de la copa leñosa y de las hojas disminuyeron. Por otro lado, la proporción de biomasa de corteza se mantuvo constante con la edad del rodal (8-10\%).

El raleo de 700 árboles ha ${ }^{-1}$, efectuado en el sexto año de edad, exportó fuera del sitio $49 \mathrm{t} \mathrm{ha}^{-1}$ de madera y aportó al suelo $6,5 \mathrm{t} \mathrm{ha}^{-1}$ de corteza, $6,7 \mathrm{t} \mathrm{ha}^{-1}$ de hojas y $8,5 \mathrm{t} \mathrm{ha}^{-1}$ de ramas totales, biomasa que se agregó al mantillo. La poda del tercio inferior de la copa de los 800 árboles residuales, equivalió a un aporte de 5,8 $\mathrm{t} \mathrm{ha}^{-1} \mathrm{de}$ biomasa leñosa (ramas, ramillas y ramas muertas) y $3,8 \mathrm{t} \mathrm{ha}^{-1}$ de hojas, que también se agregan al mantillo.

La biomasa de raíces finas fue de 5,7 $\mathrm{t} \mathrm{ha}^{-1}$ al año 4, valor que se mantuvo a los 5 años de edad, y a los 7 años de edad se registraron $3,1 \mathrm{t} \mathrm{ha}^{-1}$, en este último caso con el rodal raleado. Por ello se estimó a los 6 años 4,3 t ha ${ }^{-1}$ de raíces finas. El valor más alto de raíces gruesas se registró a la edad de 4 años con $5,5 \mathrm{t} \mathrm{ha}^{-1}$, mientras que a los 5 años fue de $3,1 \mathrm{t} \mathrm{ha}^{-1} \mathrm{y}$ a los 7 años de $4,1 \mathrm{t} \mathrm{ha}^{-1}$ (estimación a los 6 años de 3,6 t ha-1). La raíz principal experimentó un natural aumento de biomasa con la edad de la plantación y fue la que ocupó el mayor porcentaje respecto a la biomasa total de raíces (figura 1).

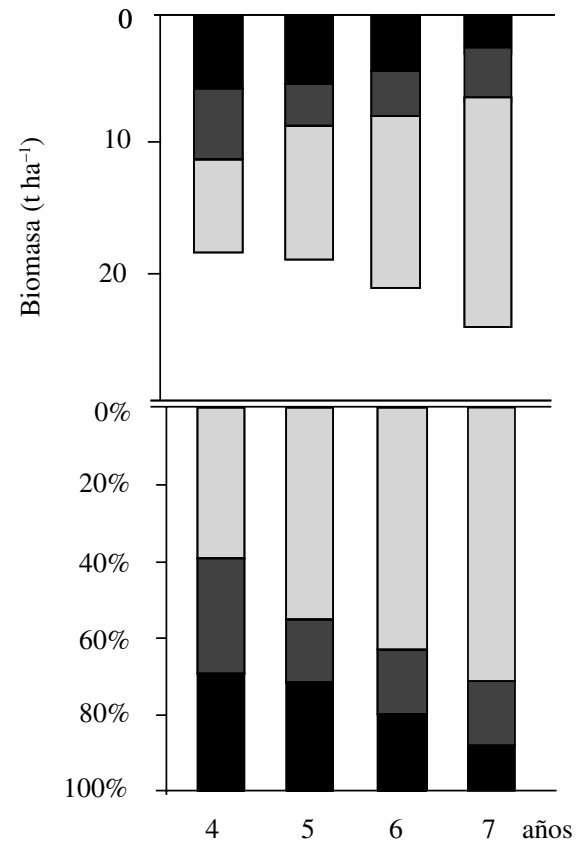

Raíces

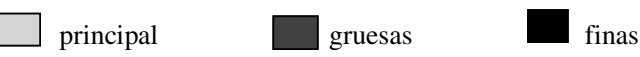

Figura 1. Biomasa de raíces y su distribución porcentual para un periodo de edad de 4-7 años en Eucalyptus nitens.

Root biomass and its distribution in percentage for an age period 4-7 years in Eucalyptus nitens.

Cuadro 5. Distribución vertical de la biomasa de la copa $\left(\mathrm{t} \mathrm{ha}^{-1}\right)$ de Eucalyptus nitens según edad.

Vertical distribution of crown biomass $\left(\mathrm{t} \mathrm{ha}^{-1}\right)$ of Eucalyptus nitens according to age.

\begin{tabular}{|c|c|c|c|c|c|c|c|c|c|c|c|}
\hline \multirow[b]{2}{*}{ Tercio } & \multicolumn{3}{|c|}{ Año 4} & \multicolumn{4}{|c|}{ Año 5} & \multicolumn{4}{|c|}{ Año 7} \\
\hline & Hoja & $\mathrm{Rama}^{\mathrm{a}}$ & R.M ${ }^{b}$ & Hoja & Ramilla & Rama & R.M & Hoja & Ramilla & Rama & R.M \\
\hline Superior & 2,1 & 1,7 & 0 & 1,4 & 1,2 & 0,3 & 0 & 1,4 & 0,7 & 0,3 & 0 \\
\hline Medio & 6,0 & 5,6 & 0 & 6,0 & 2,3 & 2,7 & 0 & 3,1 & 1,1 & 1,5 & 0 \\
\hline Inferior & 1,7 & 1,8 & 3,2 & 7,1 & 2,7 & 3,9 & 4,4 & 3,3 & 1,3 & 3,5 & 1,8 \\
\hline Total & 9,8 & 9,0 & 3,2 & 14,4 & 6,2 & 6,9 & 4,4 & 7,8 & 3,1 & 5,4 & 1,8 \\
\hline
\end{tabular}

${ }^{\mathrm{a}}$ Ramas y ramillas se consideraron en conjunto. ${ }^{\mathrm{b}}$ Ramas muertas. 
A nivel de rodal, la biomasa total de raíces correspondió a un $25 \%$ de la biomasa aérea a la edad de 4 años, disminuyendo a 17 y $13 \%$ en las edades de 5 y 6 años, respectivamente. Después del raleo, a la edad de 7 años, la biomasa de raíces representó un 22\% de la biomasa aérea, considerando que la intervención no extrajo raíces y que éstas no crecieron en los árboles cortados. Al considerar las raíces de sólo los árboles en pie (800 árboles $\mathrm{ha}^{-1}$ ), la proporción de éstas disminuyó al $17 \%$ con respecto a la biomasa aérea.

Biomasa de mantillo y hojarasca. Hasta la edad de 5 años el mantillo estuvo formado por el material originado de la caída natural de hojarasca (horizonte $\mathrm{O}$ ). A partir de los 6 años de edad se incorporaron copas y corteza debido al raleo y la poda. La acumulación del horizonte $\mathrm{O}$, sin considerar los desechos de la silvicultura, presentó fluctuaciones sin una tendencia clara con la edad, entre 5,2 y 8,6 $\mathrm{t} \mathrm{ha}^{-1}$. La biomasa de ramas y corteza en el piso del bosque producto del manejo, varió entre 12,6 y $16,9 \mathrm{t} \mathrm{ha}^{-1}$ entre los 6 y 7 años (figura 2). El horizonte $\mathrm{O}$ presentó alta variabilidad espacial $(\mathrm{CV}=16$ a $29 \%)$ con la excepción de lo ocurrido en el año 6 antes del raleo, donde se encontró un mantillo muy homogéneo en su
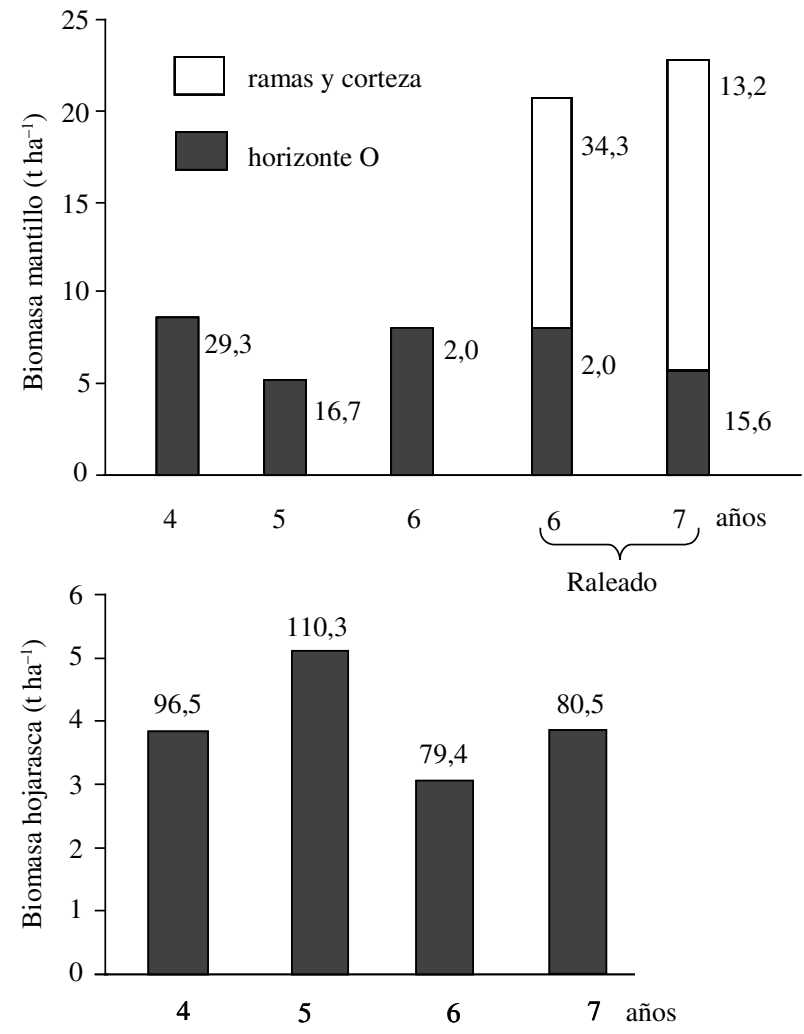

Figura 2. Biomasa del mantillo y de la caída anual de hojarasca para un periodo de 4-7 años en Eucalyptus nitens. Las cifras indican el coeficiente de variación (\%) respectivo.

Litter biomass and yearly fall of dead leaves for a period 4-7 years in Eucalyptus nitens (coefficient of variation between brackets). acumulación. Los desechos del manejo mostraron mayor variabilidad espacial $(\mathrm{CV}=18$ a $45 \%)$ que el mantillo propiamente tal, producto de la caída de hojarasca.

En el periodo de 4-6 años, antes del raleo y la poda, el rodal registró una disminución de la proporción de necromasa con respecto a la biomasa total en pie: $14 \%$ a los 4 años, $8 \%$ a los 5 años y 5\% a los 6 años de edad. Posterior al raleo, esta proporción aumentó significativamente debido a los desechos aportados al suelo por el manejo: $22 \%$ a los 6 años de edad y $19 \%$ a los 7 años.

La biomasa de la hojarasca registró su máximo valor $\left(5,1 \mathrm{t} \mathrm{ha}^{-1}\right)$ a la edad de 5 años. Las menores producciones observadas en los años 6 y 7 estuvieron influidas por el raleo y la poda (figura 2). En todos los años se observó una alta variación espacial de la caída de hojarasca, sin tendencia con la edad.

\section{DISCUSIÓN Y CONCLUSIONES}

El cultivo de Eucalyptus nitens tuvo una respuesta de alto rendimiento en los suelos rojo arcillosos de la serie Crucero, al oeste de la Depresión Intermedia, cerca de la Cordillera de la Costa, logrando producir $50 \mathrm{t} \mathrm{ha}^{-1} \mathrm{de}$ biomasa total (vuelo y raíces) en el año de mayor incremento corriente anual, a los 6 años. A través de la edad de la plantación se observó una variación natural en la acumulación de la biomasa y en la distribución porcentual de los componentes del rodal. Como es normal, la acumulación aérea siempre fue mayor que la radicular. Con respecto a la biomasa aérea, la proporción del tronco varió de un $70 \%$ a los 4 años a un $84 \%$ a los 7 años, en consecuencia, la biomasa de la copa disminuyó de un $30 \%$ a un $16 \%$ en igual período. Por otro lado, la proporción de corteza en el tronco se mantuvo casi constante con la edad de la plantación, fluctuando entre $9 \%$ y $11 \%$. La biomasa total de raíces (finas, gruesas y principal) mostró escasa variación en el período de estudio, con leves aumentos a mayor edad. En cambio, la distribución de la masa radicular experimentó cambios notorios a lo largo de los años: la proporción de raíces finas disminuyó y la de raíz principal aumentó, mientras que la proporción de raíces gruesas no cambió en los últimos tres años. Las tendencias anteriores son similares a las registradas en la literatura (Madgwick et al. 1981, Frederick et al. 1986, Attiwill y Leeper 1987, Bonomelli y Suárez 1999, Aparicio 2001, Toro 2002, Gerding et al. 2002, Muñoz 2002).

La mejor expresión de rendimiento se observó a los 6 años de edad, previo al raleo, con $18 \mathrm{t} \mathrm{ha}^{-1} \mathrm{año}^{-1} \mathrm{de}$ madera. Esto equivale a $42 \mathrm{~m}^{3} \mathrm{ha}^{-1}$ año-1, de acuerdo con los valores de densidad básica de la madera (Mutschler 2005), y a 25 metros ruma ha ${ }^{-1}$ año $^{-1}$. Este rendimiento está entre los valores altos dentro de lo observado en Chile para E. nitens (Prado y Barros 1989). Después del raleo, la plantación de E. nitens registró un buen crecimiento (16 t ha $\mathrm{haño}^{-1}$ de biomasa aérea), 
equivalente a un incremento medio anual en volumen de madera de $28 \mathrm{~m}^{3} \mathrm{ha}^{-1} \mathrm{año}^{-1}$.

El raleo significó, por una parte, el volteo de $70,8 \mathrm{t} \mathrm{ha}^{-1}$ en biomasa aérea, es decir de un $44,4 \%$ de la biomasa total acumulada a los 6 años, y por otra, exportar fuera del sitio un $30 \%$ de la biomasa aérea, en forma de madera, y reciclar un $14 \%$ de copa y corteza en el sitio. A pesar de ello, el raleo no afectó la distribución de biomasa aérea en el rodal, no obstante que esta intervención aumentó significativamente el espaciamiento entre los árboles. La distribución de biomasa en los árboles fue muy homogénea dentro del rango diamétrico observado, en cada edad analizada (Gerding et al. 2002, Saavedra 2005).

La poda, del tercio inferior de la copa de 800 árboles $\mathrm{ha}^{-1}$, reincorpora al suelo un adicional de $9 \%$ de la biomasa aérea total y es un factor importante para el reciclado del carbono y de los elementos nutritivos del sitio.

Con las cortas intermedias se produce un reciclado de elementos nutritivos que el silvicultor tiene la posibilidad de manejar con el fin de mantener la calidad del sitio. En este sentido, se debe poner especial atención al método de cosecha (sólo madera, madera con corteza, árbol completo), porque puede ser exportada una significativa cantidad de biomasa del sitio y, junto a ella, de elementos nutritivos (Belmar 2004, Geldres 2005ab).

La caída de hojarasca no mostró variación interanual significativa en sus valores promedio, aunque se aprecia una tendencia de disminución luego del raleo como respuesta a la menor densidad del rodal. En cambio, en la acumulación de mantillo se observó el efecto de las intervenciones silviculturales debido al mayor aporte de desechos como consecuencia del raleo y la poda. La hojarasca, que da origen al mantillo, alcanza su máxima caída cuando se produce el cierre de copas en el rodal, momento a partir del cual tiende a ser constante, no obstante que la producción de hojarasca es muy variable en términos espaciales (Schlatter et al. 2006). En el mismo sitio de este estudio Schlatter et al. (2006) determinaron para E. nitens una producción de hojarasca de 7 a $11 \mathrm{t} \mathrm{ha}^{-1}$ año $^{-1}$ en el período de cierre de copas y, posteriormente, fluctuó entre 4 y $6 \mathrm{t} \mathrm{ha}^{-1} \mathrm{año}^{-1}$. Estos valores son coincidentes con los observados en el presente trabajo y mayores que los encontrados en Pinus radiata D. Don en sitios equivalentes (Huber y Oyarzún 1983).

La cantidad de mantillo del rodal presentó variabilidad dentro y entre edades, producto de una caída de material de desechos naturalmente heterogénea (Moroni y Smethurst 2003) y de los aportes del raleo y de la poda. Comparativamente, la acumulación de mantillo (sin efectos de poda y raleo) en plantaciones adultas de $P$. radiata (Gerding 1991), en sitios equivalentes, es de aproximadamente tres veces lo observado en este estudio para E. nitens. No obstante, la tasa anual de caída de desechos en plantaciones de $P$. radiata es algo menor (Huber y Oyarzún 1983) que la observada en E. nitens. Entonces, la diferencia en la acumulación de mantillo se explica por la distinta tasa de descomposición de la materia orgánica que tienen estas especies. Para el presente rodal de $E$. nitens se estima una descomposición de hojas del mantillo con un ritmo de uno a dos años de acuerdo con el cuociente entre la cantidad de mantillo y la tasa anual de producción de hojarasca (Geldres 2005ab). Ello significa que $E$. nitens es muy eficiente en su dinámica de reciclado de biomasa, lo que toma especial importancia a partir del cierre de copas del rodal.

El raleo incorpora al mantillo una significativa masa de ramas y corteza, cuya descomposición es más lenta debido a su mayor composición leñosa. Esto se aprecia en la permanencia de ramas y corteza en el mantillo producto del raleo, un año después, período muy corto para su descomposición. Aún así, se espera que, por sus importantes contenidos nutritivos, este material sea descompuesto en un período no mayor a 5 años, constituyendo una significativa fuente de reabastecimiento nutritivo para el desarrollo de la plantación en el mediano plazo.

\section{AGRADECIMIENTOS}

Financiado por los proyectos FONDECYT-1010174 y Universidad Austral de Chile/DID-S-200024.

\section{REFERENCIAS}

Aparicio J. 2001. Rendimiento y biomasa de Eucalyptus nitens con alternativas nutricionales para una silvicultura sustentable en un suelo rojo arcilloso. Tesis Magíster en Ciencias. Valdivia, Chile. Facultad de Ciencias Forestales, Universidad Austral de Chile. 234 p.

Attiwill PM, GW Leeper. 1987. Forest Soils and Nutrient Cycles. In Cycles of Nutrient in Forest. Melbourne: University Press. p. 128-181.

Belmar L. 2003. Nitrógeno y calcio en la biomasa aérea de plantaciones de cinco años de Eucalyptus nitens (Dean et Maiden) Maiden con distintas fertilizaciones (comuna de Fresia, X Región). Tesis Ingeniero Forestal. Valdivia, Chile, Facultad de Ciencias Forestales, Universidad Austral de Chile. 45 p.

Bonomelli C, D Suárez. 1999. Fertilización del eucalipto. 1. Efecto sobre la acumulación de biomasa. Ciencia e Investigación Agraria 26: 1-10.

Bonomelli C, I Peña, D Suárez. 2002. Contribución de la hojas en el proceso de reciclaje de $\mathrm{N}$ y $\mathrm{P}$ en Eucalyptus $s p$. Bosque 23(1): 61-77.

CIREN (Centro de Información de Recursos Naturales, CL). 2001. Estudio agrológico X Región. Descripciones de suelos, materiales y símbolos. Tomo I. Santiago: Centro de Investigación de Recursos Naturales. 199 p.

De Freitas V, R Novais, N Barros, M Ferreira, L Dacosta.1995. Balance nutricional, eficiencia de utilización y evaluación de la fertilidad del suelo en $\mathrm{P}, \mathrm{K}, \mathrm{Ca}$ y $\mathrm{Mg}$, en parcelas con Eucalyptus saligna, en Río Grande do Sul, Brasil. In Manejo nutritivo de plantaciones forestales. Valdivia. Chile. Simposio IUFRO. p. 181-194. 
Donoso C. 1992. Ecología Forestal. El bosque y su medio ambiente. $5^{\text {a }}$ edición, Santiago, Chile. Universitaria. 369 p.

Eldridge K, J Dawidson, CH Hardwood, G Van Wyk. 1994. Eucalyptus domestication and breeding. Hong Kong, Oxford. 288 p.

FAO (Food and Agriculture Organization of the United Nations) 1981. El Eucalyptus en la repoblación forestal. Serie Montes 11. Roma. Italia. 723 p.

Frederick DJ, HAI Madgwick, MF Jurguensen, GR Oliver. 1985. Dry matter content and nutrient distribution in an age series of Eucalyptus regnans plantations in New Zealand. New Zealand Journal of Forestry Science 15(2): 58-179.

Frederick DJ, HAI Madgwick, MF Jurguensen, GR Oliver. 1986. Seasonal development of a young plantation of Eucalyptus nitens. New Zealand Journal of Forestry Science 16 (1): 78-86.

Garcinuño JL. 1995. Biomasa aérea de plantaciones jóvenes de Eucalyptus globulus (Lab.) en la comuna de Corral, Décima Región. Tesis Ingeniero Forestal. Valdivia, Chile. Facultad de Ciencias Forestales, Universidad Austral de Chile. 76 p.

Geldres E. 2005a. Dinámica de fósforo y calcio en Eucalyptus nitens de 4-7 años en un suelo Rojo Arcilloso, X Región. Tesis Magíster en Ciencias. Valdivia, Chile. Facultad de Ciencias Forestales, Universidad Austral de Chile. 95 p.

Geldres E. 2005b. Exportación de nitrógeno y potasio con la cosecha de Eucalyptus nitens. Tesis Magíster en Gestión Ambiental. Valdivia, Chile. Facultad de Ciencias Forestales, Universidad Austral de Chile. 45 p.

Gerding V. 1991. Pinus radiata-Plantagen in Zentralchile: Stanortfaktoren der Produktivität und Nahrstoffverteilung in Bestanden. Tesis Dr. Göttingen, Alemania. Forstwissenschftlichen Fachbereichs. Georg-August-Universität Göttingen. $182 \mathrm{p}$.

Gerding V, JE Schlatter. 1999. Estabilidad nutritiva de plantaciones de Pinus radiata D. Don en cinco sitios característicos de la VIII Región. Bosque 20(2): 107-115.

Gerding V, JE Schlatter, C Saavedra. 2002. Biomasa de plantaciones de Eucalyptus nitens de 5 años en un suelo rojo arcilloso con fertilización, comuna de Fresia, X Región. In Primer Congreso Chileno de Ciencias Forestales. Universidad de Chile. Santiago, Chile. 8 p.

Grove T, B Thomson, N Malajczuk. 1996. Nutritional physiology of eucalyptus: uptake, distribution and utilization. In Attiwil P, M Adams ed. Nutrition of Eucalyptus. Melbourne, Australia. CSIRO. 440 p.

Huber A, C Oyarzún. 1983. Producción de hojarasca y sus relaciones con factores meteorológicos en un bosque de Pinus radiata (D. Don). Bosque 5(1): 1-11.

Judd TS, LT Bennett, CJ Weston, PM Attiwill, PH Whiteman. 1996. The response of growth and foliar nutrients to fertilizers in young Eucalyptus globulus (Labill) plantations in Gippsland, Southeastern Australia. For. Ecol. Manage. 82: 87-101.

Leiva F. 2000. Efectos de una roca fosfórica y una cal como tratamientos base en una plantación de Eucalyptus nitens (Maiden) en un suelo rojo arcilloso de la comuna de Fresia, X Región. Tesis Ingeniero Forestal. Valdivia, Chile. Facultad de Ciencias Forestales, Universidad Austral de Chile. $82 \mathrm{p}$

Madgwick HAI, P Beets, S Gallagher. 1981. Dry matter accumulation, nutrient and energy content of the above ground portion of 4-year-old stands of Eucalyptus nitens and E. fastigata. New Zealand Journal of Forestry Science 11 (1): 53-59.

Minte E. 2004. Funciones de biomasa y volumen para $E u-$ calyptus nitens de cinco años. Tesis Ingeniero Forestal. Valdivia, Chile. Facultad de Ciencias Forestales, Universidad Austral de Chile. 82 p.

Moldenhauer F. 2003. Biomasa raíces en una plantación de 5 años de Eucalyptus nitens en un suelo rojo arcilloso con énfasis en la fertilización fosforada. Tesis Ingeniero Forestal. Valdivia, Chile. Facultad de Ciencias Forestales, Universidad Austral de Chile. 30 p.

Moroni MT, PJ Smethurst. 2003. Litterfall nitrogen and phosphorus fluxes in two Tasmanian Eucalyptus nitens plantations. Tasforests 14: 53-64.

Muñoz F. 2002. Balance nutritivo de una plantación de Eucalyptus nitens (Deane et Maiden) Maiden de 7 años de edad en suelos nadi. Tesis Ingeniero Forestal. Valdivia, Chile. Facultad de Ciencias Forestales, Universidad Austral de Chile. 108 p.

Noble A, M Herbert. 1989. Estimated nutrient removal in a short rotation Eucalyptus grandis crop on a fern wood soil. I.C.F.R. Annual Report. p. 139-150.

Mutschler M. 2005. Efecto de una fertilización sobre el plan leñoso de Eucalyptus nitens. Tesis Ingeniero Forestal. Valdivia, Chile. Facultad de Ciencias Forestales, Universidad Austral de Chile. 23 p.

Prado JA, S Barros. 1989. Eucalyptus. Principios de silvicultura y manejo. Santiago, Chile, Instituto Forestal-CORFO. $199 \mathrm{p}$.

Saavedra C. 2005. Cuantificación y distribución de biomasa aérea en una plantación de 5 años de Eucalyptus nitens con alternativas nutricionales en un suelo rojo arcilloso. Tesis Ingeniero Forestal. Valdivia, Chile. Facultad de Ciencias Forestales, Universidad Austral de Chile. 62 p.

Schlatter JE, V Gerding. 1999. Productividad en el ejemplo de seis sitios característicos de la VIII Región con Pinus radiata D. Don. Bosque 20 (1): 65-77.

Schlatter JE, V Gerding, S Calderón. 2006. Aporte de la hojarasca al ciclo biogeoquímico en plantaciones de Eucalyptus nitens, X Región, Chile. Bosque 27 (2): 115-125.

Schlatter JE, V Gerding, H Huber. 1995. Sistema de ordenamiento de la tierra; herramienta para la planificación forestal. Aplicado a la X Región. Valdivia, Chile. Universidad Austral de Chile. 93 p.

Simpson JA, RE Pegg, L Huang. 2003. An Overview of the Response of Eucalyptus to Fertilizar at Dongmen, Southern China. In Wei RP y D Xu ed. Eucalyptus Plantations. Research, Management and Development. Londres, Inglaterra, World Scientific. p. 253-268.

Staub J. 2000. Evaluación de una fertilización basada en fósforo al inicio de una plantación de Eucalyptus nitens (Deane et Maiden) Maiden en un suelo rojo arcilloso de la X Región. Tesis Ingeniero Forestal. Valdivia, Chile. Facultad de Ciencias Forestales, Universidad Austral de Chile. 94 p.

Toro J. 2002. Acumulación de calcio en plantaciones de Eucalyptus globulus y Eucalyptus nitens. Boletín de la Sociedad Chilena de la Ciencia del Suelo 18: 244-48.

Recibido: 23.08.05

Aceptado: 28.09.06 